\title{
Effect of Weather Parameters on Pearl Millet (Pennisetum glaucum L.) Cultivars under Different Sowing Windows
}

\author{
V. M. Londhe, S. G. Birajdar*, V. T. Jadhav, J. D. Jadhav and V. M. Amrutsagar \\ Zonal Agriculture Research Station, Solapur, India \\ *Corresponding author
}

\section{A B S T R A C T}

\begin{tabular}{|l|}
\hline K e y w o r d s \\
Kharif, Pearl millet, \\
$\begin{array}{l}\text { Sowing windows, } \\
\text { Yield attributes, } \\
\text { Cultivar }\end{array}$ \\
\hline Article Info \\
\hline $\begin{array}{l}\text { Accepted: } \\
10 \text { August } 2020 \\
\text { Available Online: } \\
\text { 10 September } 2020\end{array}$ \\
\hline
\end{tabular}

Present invitation entitled "Effect of weather parameters on pearl millet (Pennisetum glaucum L.) Cultivars under different sowing windows" was carried out during 2013-17 at Zonal Agricultural Research Station, Solapur, Maharashtra State (India). The experiment was conducted in split plot design with three replications. Nine treatment combinations were formed considering different cultivars viz., $\mathrm{V}_{1}$ (ICTP-8203), $\mathrm{V}_{2}$ Mahyco Hybrid and $\mathrm{V}_{3}$ Dhanashakti and sowing windows viz., $\left(\mathrm{S}_{1}\right) 2^{\text {nd }}$ fortnight of June $\left(25^{\text {th }}\right.$ June $),\left(\mathrm{S}_{2}\right)-2^{\text {nd }}$ fortnight of July $\left(27^{\text {th }}-\right.$ July) $\mathrm{S}_{3^{-}} 2^{\text {nd }}$ fortnight of August $\left(24^{\text {th }}\right.$-August $)$. Among the three pearl millet sowing window crop sown in second fortnight of July $\left(S_{2}\right)$ produced significantly higher grain yield $\left(1711.2 \mathrm{~kg} \mathrm{ha}^{-1}\right)$, biomass yield $\left(3562.6 \mathrm{~kg} \mathrm{ha}^{-1}\right)$, total monetary returns (Rs. $35145 \mathrm{ha}^{-1}$ ), CUM (319 mm), (MUE) (5.2 Kg ha $\left.{ }^{-1} \mathrm{~mm}\right)$, GDD (1958-2277 days), total dry matter $\left(901.7 \mathrm{~g} \mathrm{~m}^{-2}\right)$, LAI (1.13 ) and RUE $\left(1.98 \mathrm{~g} \mathrm{MJ}^{-1}\right)$ than other sowing dates. Among the genotypes Dhanshakti produced significantly higher grain yield $\left(1574.4 \mathrm{~kg} \mathrm{ha}^{-1}\right)$, biomass yield $\left(3192.3 \mathrm{~kg} \mathrm{ha}^{-1}\right)$ and total monetary returns (Rs. $31311 \mathrm{ha}^{-1}$ ) than other cultivars.

\section{Introduction}

Pearl millet (Pennisetum glaucum L.) formerly known as pearl, bulrush, spiked or cat-tail millet. Besides providing food and fodder, pearl millet is grown in the, hot and dry climate and also can be grown in areas like arid or semi-arid where rainfall is not sufficient as received erratic and variable amount and also have characteristics to drought tolerant warm season (Sharma et al., 2010). Rainfall, temperature and radiation are major meteorological parameters, which influence all aspects and stages of growth of the crop. It has been reported that millet has many nutritious and medical functions (Yang et al., 2001). Pearl millet grains are also used as a food for poultry and green fodder or dry kadbi for cattle. Sowing time is the most important non-monetary input influencing crop yield. Sowing at optimum time improves the productivity by providing suitable environment at all the growth stages. Upadhyay et al., (2001) have reported higher grain yield of summer pearl millet when sown on 15 march and found reduction in grain yield with delay in sowing. Time of sowing varies with the variety, agro climatic 
conditions and crop growing season. In fact, proper planting date is important for maximizing cereal grain yields (Witt, 1996) because optimum seeding dates establish healthy and vigorous plants. A significant reduction in grain yield is associated with delayed seeding for a wide range of climatic conditions (Knapp and Knapp, 1978, Dahlke et al., 1993). Lawn et al., (1993) found that differences in development of cereals sown at different times may be explained by considering an optimum temperature. It was reported that the drastic reduction in yield under delayed sowing (Ramshe et al.1986). Keeping in view of the importance the study was aimed to investigate influence of weather parameters on pearl millet (Pennisetum glaucum L.) varieties under Solapur condition.

\section{Materials and Methods}

The study was conducted at research farm of Zonal Agricultural Research Station, Solapur, Maharashtra State (India) during in year 2013-2017 in the Kharif season. The area is positioned at $75^{\circ} 65^{\prime} \mathrm{N}$ latitude $75^{\circ} 90^{\prime} \mathrm{E}$ longitude and at the altitude of 483.6 meters above the sea level. The experiment was conducted in split plot design with three replications. Nine treatment combinations were formed considering different cultivars viz., V1 (ICTP-8203), V2 Mahyco Hybrid and $\mathrm{V}_{3}$ Dhanashakti and sowing windows viz., (S1) 2nd fortnight of June ( $25^{\text {th }}$ June), $\left(\mathrm{S}_{2}\right)-2^{\text {nd }}$ fortnight of July (27 $\left.7^{\text {th }}-\mathrm{July}\right) \mathrm{S} 3-2 \mathrm{nd}$ fortnight of August (24 $4^{\text {th }}$-August). The soil comes under the vertisol (medium black) clayey loam in texture and slightly alkaline (pH-7.4) in nature and having the depth up to $90 \mathrm{~cm}$. The monsoon lasts from June to the end of September, with moderate rainfall. It has an average rainfall of about of $545 \mathrm{~mm}$ per year. The annual maximum and minimum temperature ranged between 25.0 to $43.2^{\circ} \mathrm{C}$ and 7.3 to $27.1^{0} \mathrm{C}$, respectively. The gross and net plot sizes were $15.0 \times 6.3 \mathrm{~m}^{2}$ and 10.0 $\mathrm{x} 4.5 \mathrm{~m}^{2}$, respectively. All the cultivars are were dibbled as per different sowing windows at a spacing of $45 \mathrm{~cm} \times 20 \mathrm{~cm}$.

\section{Results and Discussion}

\section{Agronomic studies}

The crop sown in second fortnight of July $\left(S_{2}\right)$ produced significantly higher grain yield (1711.2 kg ha ${ }^{-1}$ ), biomass yield (3562.6 $\mathrm{kg} \mathrm{ha}^{-1}$ ) and total monetary returns (Rs. 35145 $\mathrm{ha}^{-1}$ ) and it was found at par with crop sown in second fortnight of June $\left(S_{1}\right)$. It might be due to crop sown at second fortnight of July $\left(S_{2}\right)$ gets sufficient period for its biological and reproductive development and ultimately resulted into higher grain yield, biomass yield and total monetary returns. Whereas, adverse situation of all these parameters were experienced by delayed sown crop in $34^{\text {th }}$ and early sowing $26^{\text {th }} \mathrm{MW}$ resulted in decreased values of these yield contributing characters. Similar findings were reported by Kaushik and Gautam (1984), (Siddig et al., 2013) and (Maiti and Soto, 1990). It also indicates that crop sown at second fortnight of June $\left(S_{1}\right)$ get sufficient uniform availability of moisture during its life span helps for better yield and monetary benefits. However among the genotypes Dhanshakti produced significantly higher grain yield $\left(1574.4 \mathrm{~kg} \mathrm{ha}^{-1}\right)$, biomass yield (3192.3 $\left.\mathrm{kg} \mathrm{ha}^{-1}\right)$ and total monetary returns (Rs. $31311 \mathrm{ha}^{-1}$ ). This indicates sustainability of Dhanshakti variety over other varieties. This might be due to short duration life span of Dhanshakti than other varieties and at reproductive stage ICTP-8203 and Mahyco hybrid might be faced moisture stress condition. The results were collaborated with finding of Bashir et al., (2014) and Uzoma et al., (2010). Sustainable Yield Index (SYI) was used as an approach to evaluate the minimum yield likely to be achieved in relation to changes in sowing windows. SYI 
of July sown crop is higher (0.59) as compared early (June sown-0.39) and late sown crop (August sown-0.49). In response to change in climate treatment $\mathrm{S}_{3}$ i.e. August sown pearl millet found better in respect of SYI further, on the basis of SYI it can be included as a contingent crop for its sustainable yield of $1002.8 \mathrm{Kg} \mathrm{ha}^{-1}$ (Table 14).

\section{Meteorological studies}

The mean consumptive use of moisture (CUM) $(319 \mathrm{~mm})$ and mean moisture use efficiency (MUE) $\left(5.2 \mathrm{Kg} \mathrm{ha}^{-1} \mathrm{~mm}\right)$ was significantly higher recorded by crop sown in second fortnight of July $\left(\mathrm{S}_{2}\right)$ over rest of the sowing windows. It indicates that early and delay in sowing of crops results in recording low value of CUM and MUE. This might be due to July sown crop gets sufficient period to utilize available soil moisture along with good weather for grain production. Dhanshakti recorded highest mean value of CUM (315 $\mathrm{mm}$ ) and MUE (4.9 $\left.\mathrm{Kg} \mathrm{ha}^{-1} \mathrm{~mm}\right)$. This indicates that the Dhanshakti variety utilized the moisture most efficiently for productions of grains. The mean number of days required to attain the physiological maturity stages recorded higher in July sown crop $\left(\mathrm{S}_{2}\right)$ (Table 5). This might be due to more favorable conditions prevailed in case early sown crop and vice versa. Dhanshakti required more mean number of days to attain physiological stages (104 days) than ICTP- 8203 (97 days) and Mahyco hybrid (92 days). This indicates Dhanshakti variety required more number of days to attain physiological maturity than other varieties during kharif season under dryland conditions.

The growing degree days (GDD), the function of maximum, minimum and base temperature were presented in Table 6 . The data revealed that the growing degree days (GDD) required was $1515-1805^{\circ}$ days, $1958-2277^{\circ}$ days and
1047-1345 days for $S_{1}, S_{2}$ and $S_{3}$ sown crop. Further, it is seen that $S_{2}$ sown crop required more growing degree days to attain physiological maturity. However, among the varieties the values were higher in Dhanshakti followed by Mahyco hybrid and ICTP-8203 variety. This is due to more duration required by $\mathrm{S}_{2}$ sown crop and Dhanshakti variety. Further, it was also noticed that the early sown crop not received fairly good amount of rainfall during its growth period due to which soil moisture available was less, however, late sown crop favours due to moisture availability during flowering and grain filling stage which resulted in more duration required for maturity and good yield. The second fortnight of July $\left(\mathrm{S}_{2}\right)$ sown crop required more number of days to attain various growth stages. This is due to existence of favourable condition for crop growth and development. This is because the GDD which is function of temperature which in turn is a function of bright sunshine hours.

The mean maximum values of total dry matter were recorded by $\mathrm{S}_{2}$ sown crop i.e. $901.7 \mathrm{~g} \mathrm{~m}^{-}$ 2 over rest of the sowing windows (Table 7). Data revealed that as the delay in sowing of kharif pearl millet there is considerable reduction in mean total dry matter. Among the genotype Dhanshakti variety recorded highest values of mean total dry matter $\left(1010.6 \mathrm{~g} \mathrm{~m}^{-2}\right)$ in almost all the growth stage than the other varieties (Table 7). This indicates that the Dhanshakti utilized more efficiently moisture, light and temperature and produced maximum total dry matter by maximum solar radiation interception.

The highest mean values of LAI and RUE recorded by the crop sown in second fortnight of July (1.13 \& $1.98 \mathrm{~g} \mathrm{MJ}^{-1}$ ) (Table 8 \& 9) at 50 per cent flowering stage in almost all the sowing dates and genotypes. It was also revealed that with delayed sowing recorded low mean values of LAI and RUE. This 
indicated that the rate of conversion of light i.e. photosynthetically active radiation (PAR) was considerably high at 50 per cent flowering stage, thereafter the conversion rate was declined due to ageing of leaves. Among the sowing windows maximum mean RUE values were higher in July sown crop than late sown crop. Further, it was seen that Dhanshakti showed higher values of RUE than Mahyco and ICTP-8203 variety for conversion of light into dry matter in all the dates of sowing (Table $8 \& 9$ ).

The data in respect of mean total dry matter showed that the maximum values were recorded by Dhanshakti variety in all the windows of sowing. The July (S2) sown crop has taken maximum number of days than late sown crops to attain the different growth stages during the crop growth period. This is due to better amount of moisture available and low values of temperature during the crop growth period of July sown crops. The same trend was obtained in case of GDD this indicates that GDD is a function of bright sunshine hours which reflected into a better grain yield.

\section{Correlation and regression studies}

The weather parameter influence their contribution and performance in kharif pearl millet crop sown in different sowing windows were assessed in tenure of phasewise correlation and regression (Table 10-12).

Table.1 Pooled grain yield $\left(\mathrm{kg} \mathrm{ha}^{-1}\right)$ of kharif pearl millet as influenced by various sowing dates and varieties (2013 to 17)

\begin{tabular}{|c|c|c|c|c|c|c|c|c|}
\hline Treatment & 2013 & 2014 & 2015 & 2016 & 2017 & Mean & Sur/def $(\%)$ & SYI \\
\hline \multicolumn{9}{|l|}{ Main=3 Sowing dates } \\
\hline $\begin{array}{l}S_{1}=\text { MW } 26 \text { (June 26-July01) } \\
2^{\text {nd }} \text { fortnight of June }\end{array}$ & 2271.0 & 1452.4 & 935.4 & 1719.8 & 888.1 & 1453.3 & $\begin{array}{l}4.6 \% \text { high } \\
\text { over mean }\end{array}$ & 0.39 \\
\hline $\begin{array}{l}S_{2}=\text { MW } 30 \text { (July 23-29) } 2^{\text {nd }} \\
\text { fortnight of July }\end{array}$ & 1753.2 & 2005.5 & 1512.6 & 2185.3 & 1099.8 & 1711.3 & $\begin{array}{l}23.2 \% \text { high } \\
\text { over mean }\end{array}$ & 0.59 \\
\hline $\begin{array}{l}S_{3}=\text { MW } 35 \text { (August } 27-2 \\
\text { Sept) } 2^{\text {nd }} \text { fortnight of August }\end{array}$ & 1146.0 & 560.4 & 1268.0 & 1328.7 & 710.7 & 1002.8 & $\begin{array}{l}27.8 \% \text { less } \\
\text { over mean }\end{array}$ & 0.49 \\
\hline Mean & 1723.4 & 1339.5 & 1238.7 & 1744.6 & 899.5 & 1389.1 & & 0.59 \\
\hline \multicolumn{9}{|l|}{ Sub=Three varieties } \\
\hline$V_{1}=I C T P-8203$ & 1468.2 & 1253.8 & 1109.6 & 1598.5 & 769.1 & 1239.8 & $\begin{array}{l}10.7 \% \text { less } \\
\text { over mean }\end{array}$ & 0.57 \\
\hline$V_{2}=$ Mahyco hybrid & 1689.0 & 1288.6 & 1252.8 & 1682.7 & 852.6 & 1353.2 & $\begin{array}{l}2.6 \% \text { high } \\
\text { over mean }\end{array}$ & 0.59 \\
\hline V3= Dhanshakti & 2013.0 & 1476.0 & 1353.6 & 1952.6 & 1076.9 & 1574.4 & $\begin{array}{l}13.3 \% \text { high } \\
\text { over mean }\end{array}$ & 0.58 \\
\hline Mean & 1723.4 & 1339.5 & 1238.7 & 1744.6 & 899.5 & 1389.1 & & 0.59 \\
\hline S.E. \pm (Sowing dates) & 99.98 & 44.90 & 39.2 & 74.5 & 70.3 & 159.9 & & \\
\hline C.D. at $5 \%$ & 392.58 & 176.28 & 153.9 & 292.5 & 275.9 & 521.6 & & \\
\hline S.E. \pm (Varieties) & 70.92 & 57.30 & 57.1 & 52.0 & 48.1 & 35.7 & & \\
\hline C.D. at $5 \%$ & 218.54 & 176.57 & 175.8 & 160.3 & 148.1 & 104.2 & & \\
\hline S.E. $\pm($ SD X V) & 122.84 & 99.25 & 98.8 & 90.1 & 83.2 & 61.8 & & \\
\hline C.D. at $5 \%$ & NS & NS & NS & NS & NS & NS & & \\
\hline
\end{tabular}


Table.2 Mean Fodder yield $\left(\mathrm{kg} \mathrm{ha}^{-1}\right)$ of kharif pearl millet as influenced by various sowing dates and varieties (2013 to 17 )

\begin{tabular}{|c|c|c|c|c|c|c|c|}
\hline Treatment & 2013 & 2014 & 2015 & 2016 & 2017 & Mean & SYI \\
\hline \multicolumn{8}{|l|}{ Main=3 Sowing dates } \\
\hline $\begin{array}{l}S_{1}=\text { MW } 26 \text { (June 26-July01) } \\
2^{\text {nd }} \text { fortnight of June }\end{array}$ & 3126.9 & 3157.2 & 2047.4 & 3046.7 & 1828.0 & 2641.3 & 0.63 \\
\hline $\begin{array}{l}S_{2}=\text { MW } 30 \text { (July. 23-29) } 2^{\text {nd }} \text { fortnight } \\
\text { of July }\end{array}$ & 4937.7 & 4671.2 & 2775.2 & 3393.2 & 2035.9 & 3562.6 & 0.47 \\
\hline $\begin{array}{l}S_{3}=M W 35 \text { (August 27-2 Sept) } \\
2^{\text {nd }} \text { fortnight of August }\end{array}$ & 2767.9 & 1493.5 & 2634.7 & 2665.4 & 1599.3 & 2232.2 & 0.58 \\
\hline Mean & 3610.9 & 3107.3 & 2485.8 & 3035.1 & 1821.1 & 2812.0 & 0.59 \\
\hline \multicolumn{8}{|l|}{ Sub $=3$ varieties } \\
\hline V1= ICTP-8203 & 3313.9 & 3029.7 & 2174.3 & 2723.7 & 1634.2 & 2575.2 & 0.57 \\
\hline$V_{2}=$ Mahyco hybrid & 3457.2 & 2674.9 & 2435.5 & 2984.8 & 1790.9 & 2668.7 & 0.59 \\
\hline V3 = Dhanshakti & 4061.5 & 3617.2 & 2847.5 & 3396.9 & 2038.1 & 3192.3 & 0.59 \\
\hline Mean & 3610.9 & 3107.3 & 2485.8 & 3035.1 & 1821.1 & 2812.0 & 0.59 \\
\hline S.E. \pm (Sowing dates) & 115.62 & 99.51 & 109.7 & 109.7 & 65.8 & 307.1 & \\
\hline C.D. at $5 \%$ & 453.98 & 390.72 & 430.6 & 430.6 & 258.4 & 1001.5 & \\
\hline S.E. \pm (Varieties) & 145.81 & 156.22 & 120.7 & 120.7 & 72.4 & 81.1 & \\
\hline C.D. at $5 \%$ & 449.28 & 481.35 & 371.8 & 371.8 & 223.1 & 236.7 & \\
\hline S.E. $\pm($ SD X V) & 252.55 & 270.57 & 209.0 & 209.0 & 125.4 & 140.5 & \\
\hline C.D. at $5 \%$ & NS & NS & NS & NS & NS & NS & \\
\hline
\end{tabular}

Table.3 Mean total monetary returns $\left(\mathrm{Rs} \mathrm{ha}^{-1}\right)$ of kharif pearl millet as influenced by various sowing dates and varieties (2013 to 17 )

\begin{tabular}{|c|c|c|c|c|c|c|c|}
\hline Treatment & 2013 & 2014 & 2015 & 2016 & 2017 & Mean & SYI \\
\hline \multicolumn{8}{|l|}{ Main=3 Sowing dates } \\
\hline $\begin{array}{l}S_{1}=\text { MW } 26 \text { (June 26-July01) } \\
2^{\text {nd }} \text { fortnight of June }\end{array}$ & 29236 & 28100 & 23074 & 31891 & 22332 & 26927 & 0.72 \\
\hline $\begin{array}{l}S_{2}=\text { MW } 30\left(\text { July. 23-29) } 2^{\text {nd }} \text { fortnight of }\right. \\
\text { July }\end{array}$ & 39201 & 39426 & 30445 & 39566 & 27086 & 35145 & 0.74 \\
\hline $\begin{array}{l}S_{3}=M W 35 \text { (August } 27-2 \text { Sept) } \\
2^{\text {nd }} \text { fortnight of August }\end{array}$ & 21291 & 11393 & 26400 & 25261 & 18212 & 20511 & 0.55 \\
\hline Mean & 29909 & 26306 & 26640 & 32239 & 22543 & 27528 & 0.74 \\
\hline \multicolumn{8}{|l|}{ Sub $=3$ varieties } \\
\hline$V_{1}=I C T P-8203$ & 25099 & 24241 & 24092 & 29425 & 19467 & 24465 & 0.71 \\
\hline $\mathrm{V}_{2}=$ Mahyco hybrid & 29259 & 25304 & 26733 & 31210 & 21529 & 26807 & 0.74 \\
\hline$V_{3}=$ Dhanshakti & 35369 & 29374 & 29094 & 36082 & 26634 & 31311 & 0.75 \\
\hline Mean & 29909 & 26306 & 26640 & 32239 & 22543 & 27528 & 0.74 \\
\hline S.E. \pm (Sowing dates) & 1437.17 & 815.3 & 670.5 & 1290.0 & 1522.6 & 2179.1 & \\
\hline C.D. at $5 \%$ & 5643.03 & 3201.2 & 2632.6 & 5065.2 & 5978.4 & 7106.6 & \\
\hline S.E. \pm (Varieties) & 867.80 & 1138.0 & 737.5 & 725.3 & 993.0 & 592.7 & \\
\hline C.D. at $5 \%$ & 2673.96 & 3506.5 & 2272.4 & 2234.9 & 3059.7 & 1729.9 & \\
\hline S.E. $\pm($ SD X V) & 1503.07 & 1971.1 & 1277.3 & 1256.3 & 1719.9 & 1026.6 & \\
\hline C.D. at $5 \%$ & NS & NS & NS & NS & NS & NS & \\
\hline
\end{tabular}


Table.4 Mean CUM and MUE as influenced by sowing windows and varieties in Kharif pearl millet (2013 to 17)

\begin{tabular}{|c|c|c|c|}
\hline Treatment & GY $\left(\mathbf{k g ~ h a}^{\mathbf{- 1}}\right)$ & CUM $(\mathbf{m m})$ & MUE $\left(\mathbf{k g ~ h a}^{-\mathbf{1}} \mathbf{~ m m}\right)$ \\
\hline $\mathbf{S}_{\mathbf{1}} \mathbf{V}_{\mathbf{1}}$ & 1358.9 & 290 & 4.7 \\
\hline $\mathbf{S}_{\mathbf{1}} \mathbf{V}_{\mathbf{2}}$ & 1448.5 & 304 & 4.8 \\
\hline $\mathbf{S}_{\mathbf{1}} \mathbf{V}_{\mathbf{3}}$ & 1552.7 & 314 & 4.9 \\
\hline $\mathbf{S}_{\mathbf{2}} \mathbf{V}_{\mathbf{1}}$ & 1542.9 & 317 & 4.9 \\
\hline $\mathbf{S}_{\mathbf{2}} \mathbf{V}_{\mathbf{2}}$ & 1631.9 & 308 & 5.3 \\
\hline $\mathbf{S}_{\mathbf{2}} \mathbf{V}_{\mathbf{3}}$ & 1959.1 & 332 & 5.9 \\
\hline $\mathbf{S}_{\mathbf{3}} \mathbf{V}_{\mathbf{1}}$ & 817.7 & 270 & 3.0 \\
\hline $\mathbf{S}_{\mathbf{3}} \mathbf{V}_{\mathbf{2}}$ & 979.2 & 285 & 3.4 \\
\hline $\mathbf{S}_{\mathbf{3}} \mathbf{V}_{\mathbf{3}}$ & 1211.4 & 300 & 4.0 \\
\hline
\end{tabular}

Table.5 Mean number of days required to attain phenological stages as influenced by sowing windows and varieties in kharif pearl millet (2013 to 17)

\begin{tabular}{|c|c|c|c|c|c|c|c|c|}
\hline $\begin{array}{c}\text { Sowing } \\
\text { Time }\end{array}$ & \multicolumn{7}{|c|}{ Phenological stage } \\
\hline & Emer. & $\mathbf{3}$ leaf & PI & Flag leaf & $\begin{array}{c}\mathbf{5 0} \% \\
\text { flowering }\end{array}$ & $\begin{array}{c}\text { Soft } \\
\text { dough }\end{array}$ & $\begin{array}{c}\text { Hard } \\
\text { dough }\end{array}$ & $\begin{array}{c}\text { Phy. } \\
\text { Maturity }\end{array}$ \\
\hline $\mathbf{S}_{\mathbf{1}} \mathbf{V}_{\mathbf{1}}$ & 8 & 7 & 18 & 22 & 11 & 13 & 14 & 9 \\
\hline Cumulative & 8 & 15 & 33 & 55 & 66 & 79 & 93 & 102 \\
\hline $\mathbf{S}_{\mathbf{1}} \mathbf{V}_{\mathbf{2}}$ & 8 & 8 & 15 & 19 & 12 & 14 & 11 & 10 \\
\hline Cumulative & 8 & 16 & 31 & 50 & 62 & 76 & 87 & 97 \\
\hline $\mathbf{S}_{\mathbf{1}} \mathbf{V}_{\mathbf{3}}$ & 8 & 8 & 18 & 22 & 11 & 17 & 14 & 9 \\
\hline Cumulative & 8 & 16 & 34 & 56 & 67 & 84 & 98 & 107 \\
\hline $\mathbf{S}_{\mathbf{2}} \mathbf{V}_{\mathbf{1}}$ & 8 & 7 & 15 & 23 & 15 & 13 & 12 & 10 \\
\hline Cumulative & 8 & 15 & 30 & 53 & 68 & 81 & 93 & 103 \\
\hline $\mathbf{S}_{\mathbf{2}} \mathbf{V}_{\mathbf{2}}$ & 6 & 4 & 14 & 22 & 14 & 14 & 13 & 09 \\
\hline Cumulative & 6 & 10 & 24 & 46 & 60 & 74 & 87 & 96 \\
\hline $\mathbf{S}_{\mathbf{2}} \mathbf{V}_{\mathbf{3}}$ & 8 & 8 & 16 & 27 & 15 & 16 & 14 & 09 \\
\hline Cumulative & 8 & 14 & 30 & 57 & 72 & 88 & 102 & 111 \\
\hline $\mathbf{S}_{\mathbf{3}} \mathbf{V}_{\mathbf{1}}$ & 6 & 5 & 14 & 22 & 10 & 10 & 11 & 9 \\
\hline Cumulative & 6 & 11 & 25 & 47 & 57 & 67 & 78 & 87 \\
\hline $\mathbf{S}_{\mathbf{3}} \mathbf{V}_{\mathbf{2}}$ & 5 & 5 & 15 & 19 & 11 & 8 & 11 & 9 \\
\hline Cumulative & 5 & 10 & 25 & 44 & 55 & 63 & 74 & 83 \\
\hline $\mathbf{S}_{\mathbf{3}} \mathbf{V}_{\mathbf{3}}$ & 6 & 6 & 16 & 23 & 12 & 11 & 12 & 7 \\
\hline Cumulative & 6 & 12 & 28 & 51 & 63 & 74 & 86 & 93 \\
\hline
\end{tabular}


Table.6 Mean growing degree days $\left({ }^{0} \mathrm{C}\right)$ required to attain growth stages as influenced by sowing windows and varieties in kharif pearl millet (2013-17)

\begin{tabular}{|c|c|c|c|c|c|c|c|c|}
\hline $\begin{array}{c}\text { Sowing } \\
\text { Time }\end{array}$ & \multicolumn{9}{|c|}{ Emer. } & $\mathbf{3}$ leaf & PI & Flag leaf & $\begin{array}{c}\mathbf{5 0} \text { \% } \\
\text { flowering }\end{array}$ & $\begin{array}{c}\text { Soft } \\
\text { dough }\end{array}$ & $\begin{array}{c}\text { Hard } \\
\text { dough }\end{array}$ & $\begin{array}{c}\text { Phy. } \\
\text { Maturity }\end{array}$ \\
\hline $\mathbf{S}_{\mathbf{1}} \mathbf{V}_{\mathbf{1}}$ & 52 & 41 & 309 & 325 & 235 & 201 & 194 & 180 \\
\hline Cumulative & 52 & 90 & 395 & 717 & 949 & 1147 & 1338 & 1515 \\
\hline $\mathbf{S}_{\mathbf{1}} \mathbf{V}_{\mathbf{2}}$ & 60 & 56 & 368 & 283 & 260 & 219 & 233 & 221 \\
\hline Cumulative & 60 & 113 & 478 & 758 & 1015 & 1231 & 1461 & 1679 \\
\hline $\mathbf{S}_{\mathbf{1}} \mathbf{V}_{\mathbf{3}}$ & 58 & 45 & 416 & 378 & 276 & 210 & 212 & 232 \\
\hline Cumulative & 58 & 100 & 513 & 888 & 1161 & 1367 & 1576 & 1805 \\
\hline $\mathbf{S}_{\mathbf{2}} \mathbf{V}_{\mathbf{1}}$ & 69 & 50 & 453 & 383 & 291 & 255 & 232 & 248 \\
\hline Cumulative & 69 & 116 & 565 & 945 & 1232 & 1484 & 1713 & 1958 \\
\hline $\mathbf{S}_{\mathbf{2}} \mathbf{V}_{\mathbf{2}}$ & 53 & 49 & 417 & 362 & 279 & 205 & 216 & 185 \\
\hline Cumulative & 53 & 98 & 512 & 870 & 1146 & 1348 & 1561 & 1742 \\
\hline $\mathbf{S}_{\mathbf{2}} \mathbf{V}_{\mathbf{3}}$ & 75 & 64 & 539 & 449 & 314 & 284 & 267 & 308 \\
\hline Cumulative & 75 & 135 & 671 & 1117 & 1428 & 1709 & 1972 & 2277 \\
\hline $\mathbf{S}_{\mathbf{3}} \mathbf{V}_{\mathbf{1}}$ & 36 & 31 & 254 & 248 & 106 & 113 & 112 & 161 \\
\hline Cumulative & 36 & 65 & 316 & 563 & 666 & 778 & 888 & 1047 \\
\hline $\mathbf{S}_{\mathbf{3}} \mathbf{V}_{\mathbf{2}}$ & 49 & 36 & 279 & 259 & 144 & 125 & 112 & 163 \\
\hline Cumulative & 49 & 82 & 359 & 616 & 757 & 880 & 990 & 1151 \\
\hline $\mathbf{S}_{\mathbf{3}} \mathbf{V}_{\mathbf{3}}$ & 52 & 38 & 308 & 298 & 153 & 183 & 143 & 186 \\
\hline Cumulative & 52 & 87 & 393 & 689 & 840 & 1021 & 1161 & 1345 \\
\hline
\end{tabular}

Table.7 Mean periodical dry matter $\left(\mathrm{g} \mathrm{m}^{-2}\right)$ and its partitioning into different parts of kharif pearl millet as influenced by sowing windows and varieties (2013 to 17)

\begin{tabular}{|c|c|c|c|c|c|c|}
\hline $\begin{array}{c}\text { Sowing } \\
\text { Date }\end{array}$ & Emer. & PI & $\mathbf{5 0 \%}$ flow. & Soft Dough & Hard Dough & Phy. Maturity \\
\hline & & & \multicolumn{2}{|c|}{ Total } & \\
\hline S1V1 & 0.7 & 146.7 & 353.4 & 708.5 & 920.5 & 762.9 \\
\hline S1V2 & 0.7 & 112.9 & 319.6 & 712.4 & 924.4 & 769.4 \\
\hline S1V3 & 0.9 & 83.2 & 289.9 & 746.4 & 958.4 & 793.0 \\
\hline S2V1 & 0.9 & 168.8 & 375.5 & 790.7 & 1032.7 & 872.7 \\
\hline S2V2 & 0.9 & 147.8 & 354.5 & 800.0 & 962.3 & 902.4 \\
\hline S2V3 & 0.8 & 181.2 & 387.9 & 829.9 & 989.7 & 930.1 \\
\hline S3V1 & 0.9 & 149.1 & 355.8 & 749.6 & 988.6 & 829.9 \\
\hline S3V2 & 0.9 & 207.9 & 414.6 & 772.5 & 1014.5 & 854.5 \\
\hline S3V3 & 1.0 & 89.2 & 295.9 & 788.4 & 1050.4 & 890.4 \\
\hline
\end{tabular}


Table.8 Mean leaf area index as influenced by sowing windows and varieties in Kharif pearl millet (2013 to 17)

\begin{tabular}{|c|c|c|c|c|c|c|c|}
\hline Sowing & \multicolumn{9}{|c|}{ Phenological stage } \\
\hline Time & Emer. & PI & Flag leaf & $\begin{array}{c}\mathbf{5 0} \% \\
\text { flowering }\end{array}$ & $\begin{array}{c}\text { Soft } \\
\text { dough }\end{array}$ & $\begin{array}{c}\text { Hard } \\
\text { Dough }\end{array}$ & $\begin{array}{c}\text { Phy. } \\
\text { maturity }\end{array}$ \\
\hline S1V1 & 0.03 & 0.39 & 0.72 & 1.00 & 1.09 & 0.77 & 0.39 \\
\hline S1V2 & 0.03 & 0.27 & 0.56 & 0.84 & 0.93 & 0.47 & 0.34 \\
\hline S1V3 & 0.03 & 0.31 & 0.65 & 0.93 & 1.02 & 0.55 & 0.25 \\
\hline S2V1 & 0.06 & 0.56 & 1.14 & 1.42 & 1.51 & 1.22 & 0.91 \\
\hline S2V2 & 0.04 & 0.38 & 0.92 & 1.20 & 1.29 & 0.61 & 0.52 \\
\hline S2V3 & 0.05 & 0.49 & 1.06 & 1.34 & 1.43 & 1.10 & 0.77 \\
\hline S3V1 & 0.04 & 0.34 & 0.78 & 1.06 & 1.15 & 0.67 & 0.57 \\
\hline S3V2 & 0.05 & 0.31 & 0.72 & 1.00 & 1.09 & 0.90 & 0.47 \\
\hline S3V3 & 0.04 & 0.41 & 0.72 & 1.00 & 1.09 & 0.92 & 0.47 \\
\hline
\end{tabular}

Table.9 Mean radiation use efficiency $\left(\mathrm{g} \mathrm{MJ}^{-1}\right)$ as influenced by sowing windows and varieties in kharif pearl millet (2013 to 17)

\begin{tabular}{|l|c|c|c|c|c|c|c|}
\hline Sowing & \multicolumn{7}{|c|}{ Phenological stage } \\
\hline Time & Emer. & PI & $\begin{array}{c}\text { Flag } \\
\text { leaf }\end{array}$ & $\begin{array}{c}\mathbf{5 0} \% \\
\text { flowering }\end{array}$ & $\begin{array}{c}\text { Soft } \\
\text { dough }\end{array}$ & $\begin{array}{c}\text { Hard } \\
\text { Dough }\end{array}$ & $\begin{array}{c}\text { Phy. } \\
\text { maturity }\end{array}$ \\
\hline $\mathrm{S}_{1} \mathrm{~V}_{1}$ & 0.08 & 0.21 & 0.84 & 0.95 & 0.93 & 0.83 & 0.72 \\
\hline $\mathrm{S}_{1} \mathrm{~V}_{2}$ & 0.06 & 0.08 & 0.62 & 1.89 & 0.62 & 0.50 & 0.37 \\
\hline $\mathrm{S}_{1} \mathrm{~V}_{3}$ & 0.06 & 0.14 & 0.74 & 1.68 & 0.89 & 0.73 & 0.56 \\
\hline $\mathrm{S}_{2} \mathrm{~V}_{1}$ & 0.13 & 0.25 & 1.40 & 1.84 & 1.55 & 1.24 & 1.14 \\
\hline $\mathrm{S}_{2} \mathrm{~V}_{2}$ & 0.10 & 0.20 & 0.82 & 1.85 & 1.31 & 1.16 & 0.95 \\
\hline $\mathrm{S}_{2} \mathrm{~V}_{3}$ & 0.10 & 0.22 & 1.45 & 2.24 & 1.62 & 1.38 & 1.12 \\
\hline $\mathrm{S}_{3} \mathrm{~V}_{1}$ & 0.13 & 0.26 & 1.26 & 1.11 & 1.41 & 1.19 & 1.10 \\
\hline $\mathrm{S}_{3} \mathrm{~V}_{2}$ & 0.13 & 0.44 & 1.15 & 1.31 & 1.04 & 1.08 & 0.67 \\
\hline $\mathrm{S}_{3} \mathrm{~V}_{3}$ & 0.32 & 0.32 & 0.87 & 1.44 & 1.24 & 1.13 & 0.79 \\
\hline
\end{tabular}

Table.10 Correlation coefficient between grain yield and different weather parameters during different phenophases of kharif pearl millet

\begin{tabular}{|l|c|c|c|c|c|c|c|c|}
\hline Phenophase & $\begin{array}{c}\text { MAXT } \\
\mathbf{(} \mathbf{C})\end{array}$ & $\begin{array}{c}\text { MINT } \\
\mathbf{( \mathbf { C } )}\end{array}$ & $\begin{array}{c}\text { RH1 } \\
\mathbf{( \% )}\end{array}$ & $\begin{array}{c}\text { RH2 } \\
\mathbf{( \% )}\end{array}$ & $\begin{array}{c}\text { WS } \\
\mathbf{( k m p h )}\end{array}$ & $\begin{array}{c}\text { RF } \\
(\mathbf{m m} / \mathbf{d a y})\end{array}$ & $\begin{array}{c}\text { SS } \\
\text { (hrs/day) }\end{array}$ & $\begin{array}{c}\text { EVP } \\
(\mathbf{m m} / \mathbf{d a y})\end{array}$ \\
\hline P1 & -0.270 & 0.220 & -0.308 & 0.355 & $0.805^{* *}$ & -0.147 & -0.471 & 0.411 \\
\hline P2 & -0.148 & 0.246 & -0.461 & -0.250 & 0.607 & -0.095 & -0.314 & 0.435 \\
\hline P3 & $0.746^{*}$ & 0.419 & $-0.859^{* *}$ & $-0.671^{*}$ & 0.451 & $-0.768^{*}$ & -0.025 & 0.525 \\
\hline P4 & -0.608 & 0.584 & 0.641 & $0.758^{*}$ & 0.400 & 0.334 & -0.613 & $0.668^{*}$ \\
\hline P5 & -0.438 & $0.678^{*}$ & $0.771^{*}$ & $0.683^{*}$ & 0.170 & $0.823^{* *}$ & -0.475 & $0.748^{*}$ \\
\hline P6 & 0.353 & 0.398 & 0.328 & 0.159 & 0.080 & 0.250 & 0.066 & $0.836^{* *}$ \\
\hline P7 & 0.326 & 0.303 & 0.105 & 0.239 & 0.012 & 0.028 & -0.125 & 0.213 \\
\hline P8 & 0.153 & 0.176 & -0.043 & 0.082 & 0.264 & 0.159 & 0.022 & 0.396 \\
\hline
\end{tabular}


Table.11 Stepwise multiple regression of different weather parameters with yield of kharif pearl millet at 50\% flowering stage (2013 to 2017)

\begin{tabular}{|l|l|c|l|}
\hline Sr. No. & Weather parameter & Regression coefficient & $\mathbf{R}^{\mathbf{2}}$ \\
\hline $\mathbf{1}$ & Intercept & -101.38 & $\mathbf{0 . 8 1}$ \\
\hline $\mathbf{2}$ & Minimum Temperature $\left(\mathrm{T}_{\min }\right)$ & -0.21 & \\
\hline $\mathbf{3}$ & Relative Humidity $(\mathrm{RH}-1)$ & 2.49 \\
\hline $\mathbf{4}$ & Relative Humidity $(\mathrm{RH}-2)$ & -1.34 & \\
\hline $\mathbf{5}$ & Rainfall (RF) & $\mathbf{0 . 0 8}$ & \\
\hline
\end{tabular}

Table.12 Observed and predicted yield by using linear regression equations

\begin{tabular}{|c|c|c|c|c|}
\hline Treatment & $\begin{array}{l}\text { Actual } \\
\text { Yield }\end{array}$ & $\begin{array}{l}\text { Predicted } \\
\text { Yield }\end{array}$ & Residuals & $\begin{array}{l}\text { Standardized } \\
\text { residual }\end{array}$ \\
\hline \multicolumn{5}{|l|}{ Main treatment - Sowing time } \\
\hline $\begin{array}{l}S_{1}=M W 26 \text { (June 26-July01) } \\
2^{\text {nd }} \text { fortnight of June }\end{array}$ & 1533.4 & 1501.5 & -31.9 & -0.63 \\
\hline $\begin{array}{l}S_{2}=\text { MW } 30 \text { (July. 23-29) } 2^{\text {nd }} \text { fortnight } \\
\text { of July }\end{array}$ & 1757.0 & 1829.2 & 72.2 & 1.15 \\
\hline $\begin{array}{l}S_{3}=M W 35 \text { (August 27-Sept 2) } \\
2^{\text {nd }} \text { fortnight of August }\end{array}$ & 1034.2 & 1008.0 & -26.2 & -0.53 \\
\hline \multicolumn{5}{|l|}{ Sub treatment - variety } \\
\hline$V_{1}=I C T P-8203$ & 1418.6 & 1298.6 & -120.0 & -1.15 \\
\hline$V_{2}=$ Mahyco hybrid & 1365.9 & 1425.3 & 59.4 & 0.51 \\
\hline$V_{3}=$ Dhanshakti & 1540.1 & 1614.8 & 74.7 & 0.65 \\
\hline $\begin{array}{ll}\sqrt{Y} \text { ield }=-101.38+\left(-0.21 \times \mathrm{T}_{\min }\right)+(2.49 \times \mathrm{RH}-1)+ \\
\text { Standard Residual }>3 \text { is outleter, } \\
\mathrm{T}_{\min } \\
\mathrm{RH}-1 & =\mathrm{N} \\
\mathrm{RH}-2 & =\text { Even } \\
\mathrm{RF} & \end{array}$ & $\begin{array}{l}1.34 \times \mathrm{RH}-2 \text {, } \\
\text { Temperat } \\
\text { g relative } \mathrm{h} \\
\text { g relative hu } \\
\text { Rainfall ( } \mathrm{n}\end{array}$ & $\begin{array}{l}(0.08 \times \mathrm{RF}) \\
\text { nidity }(\%) \\
\text { nidity }(\%)\end{array}$ & & \\
\hline
\end{tabular}

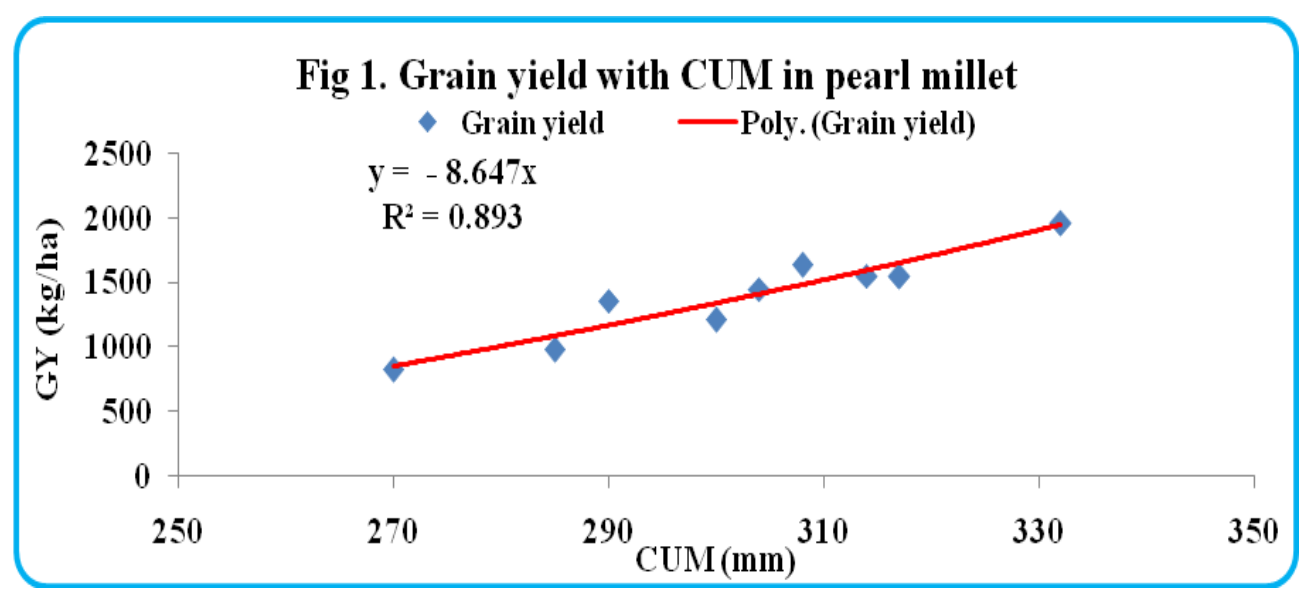



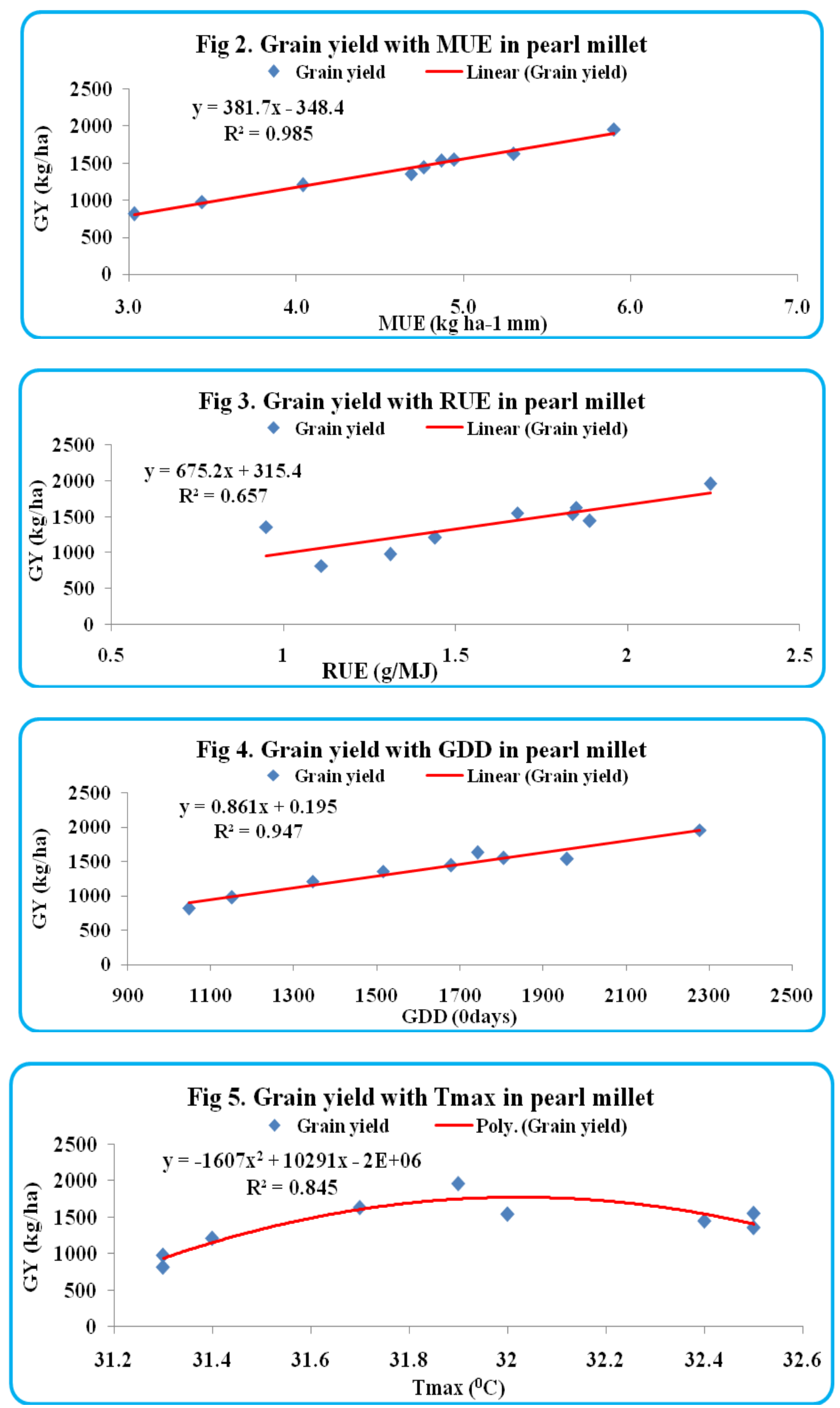


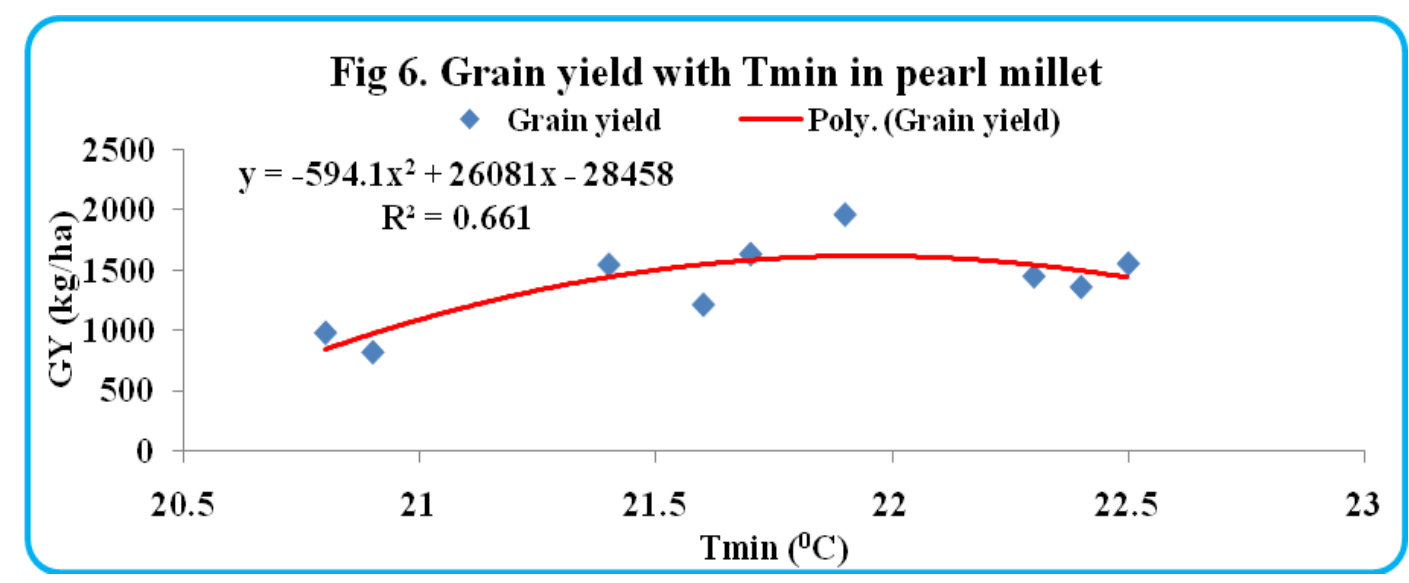

The influence of weather parameter and agrometeorological indices on performance of kharif pearl millet crop sown at different windows with different varieties were assured in terms of phase-wise correlation of grain yield with weather parameters. It is revealed that the wind speed had significant positive correlation at emergence phase (P1). Tmax had significant positive influence and RH-1, RH-2 and RF has significant negative influence at panicle initiation phase (P3). RH2 and Epan had significant positive correlation at flag leaf stage (P4). Significant positive correlation was found with $\mathrm{T}_{\min }, \mathrm{RH}-$ 1, RH-2, RF and $\mathrm{E}_{\mathrm{pan}}$ at $50 \%$ flowering stage (P5) while at soft dough stage Epan had significant positive association with grain yield. Significant negative association with grain yield by $\mathrm{T}_{\min }, \mathrm{RH}-1, \mathrm{RH}-2$ and $\mathrm{RF}$ at panicle initiation phase $(\mathrm{P} 3)$ indicates that at early growth stages kharif pearl millet not favour moisture stress condition. Significant positive association with grain yield at $50 \%$ flowering stage by $\mathrm{T}_{\min }, \mathrm{RH}-1, \mathrm{RH}-2, \mathrm{RF}$ and $\mathrm{E}_{\mathrm{pan}}$ indicates kharif pearl millet responds well to available moisture and low temperature conditions. It is revealed that panicle initiation stage (P3) and 50\% flowering stage (P5) are more crucial growth stages to contribute grain production.

It is observed that the significantly positive correlation (Table 10) of weather parameters namely Tmin, RH-1, RH-2 and RF with grain yield at 50\% flowering phase. The predicted grain yield and actual pooled grain yield is presented in Table 12. The regression equation is developed by using this weather parameters i.e. $\sqrt{ }$ Yield $=-101.38+(-$ $0.21 \times$ Tmin $)+(2.49 \times \mathrm{RH}-1)+(-1.34 \times \mathrm{RH}-2)+$ $(0.08 \times \mathrm{RF})$. This equation is helpful to predict grain yield after completion of $50 \%$ flowering phase (P5) (Table 11 and 12).

The consumptive use of moisture (CUM) during total growth period of kharif pearl millet Fig. 1 showed a polynomial relationship with grain yield $\left(\mathrm{y}=0.0437 \mathrm{x}^{2}\right.$ $8.6471 \times \mathrm{R}^{2}=0.89$ ). The CUM of $310 \mathrm{~mm}$ was found to be optimum for getting higher grain yield. The moisture use efficiency (MUE) during total growth period of kharif pearl millet Fig. 2 showed a linear relationship with grain yield $\left(\mathrm{y}=381.76 \mathrm{x}-348.47 \mathrm{R}^{2}=0.98\right)$. The MUE of 4.5 to $5.5 \mathrm{~kg}$ ha $\mathrm{mm}^{-1}$ was found to be optimum for getting higher grain yield. The RUE studies depicted in Fig. 3 showed linear relationship with grain yield.

This indicated that radiation interception is directly related with grain yield $\left(y=675.25 x+315.49 R^{2}=0.66\right)$. The figure showed that if RUE increases from 1.5 to 1.9 $\mathrm{g} \mathrm{mj}^{-1}$ it increases the yield from 07 to $14 \mathrm{q}$ $\mathrm{ha}^{-1}$. This indicated that every increase of 0.1 $\mathrm{gmj}^{-1}$ of energy there is increase of $0.94 \mathrm{q} \mathrm{ha}^{-1}$ of grain yield of pearl millet. 
The GDD was correlated with the grain yield of pearl millet and depicted in Fig. 4. It showed a linear relationship with grain yield $\left(y=0.861 x+0.1952 R^{2}=0.95\right)$. This indicated that with increase of GDD there was increase in grain yield upto 1800 GDD. The $\mathrm{T}_{\max }$ was correlated with the grain yield of pearl millet and depicted in Fig. 5. It showed a polynomial relationship with grain yield $(\mathrm{y}=-$ $\left.1607 \mathrm{x}^{2}+102918 \mathrm{x}-2 \mathrm{E}+06 \mathrm{R}^{2}=0.85\right)$. This indicated that with increase of $\mathrm{T}_{\max }$ there was increase in grain yield upto $31.9^{\circ} \mathrm{C}$ and later on yield decrease with increase in $\mathrm{T}_{\max }$. The $\mathrm{T}_{\text {min }}$ was correlated with the grain yield of pearl millet and depicted in Fig. 6. It showed a polynomial relationship with grain yield $\left(\mathrm{y}=-594.18 \mathrm{x}^{2}+26081 \mathrm{x}-284580 \mathrm{R}^{2}=0.66\right)$. This indicated that with increase of $\mathrm{T}$ min there was increase in grain yield upto $21.9^{\circ} \mathrm{C}$ later on yield decrease with increase in $\mathrm{T}_{\min }$

\section{References}

Bashir, M.M.H., Yagoub, S.O. and Ahmed Mohammed, S. A.2015. Effect of different sowing dates on growth and yield of three pearl millet (Pennisetum glaucum (L.)R. Br) cultivars in West Darfur Sudan. International J. Pl. \& Soil Sci.5(4):191-200.

Dahlke, B.J., Oplinger, E.S., Gaska, J.M. and Martinka, M.J. 1993. Influence of planting date and seedling rate on winter wheat grain yield and yield components. J. Prod. Agric. 6: 408-414.

Kaushik, S.K. and Gautam, R.C. 1984. Effect of varying dates of planting and row spacing on yield of pearl millet varieties under rainfed conditions. Indian $J$. Agron. 29 (3): 480-484.

Knapp, W.R. and Knapp, J. S. 1978. Response of winter wheat to date of planting and fall fertilization. Agron. J. 70: 1048-1053.

Lawn, R.J., Summer field, R.J., Ellis, R.H., Roberts, E.H., Chay, P.M., Brouwer,
J.B., Rose, J.L. and Yeates, S.J. 1993. Towards the reliable prediction of time to flowering in six annual crops. VI. Applications in crop improvement. Exptt. Agric., 31: 89-108.

Maiti R.K., Soto G.G.1990. Effect of four sowing date environments on growth, development and yield potentials of 15 pearl millet cultivars (Pennisetum americanum L Leeke) during autumn winter seasons in Marin, N.L., Mexico. Arpn. J. 1999; 3(3): 30-37.

Ramshe, D.G., Patil, B.R. and Umrani, N.K. 1986. Effects of planting time and nitrogen on yield of pearl millet varieties. J. Maharashtra agric. Univ., 11(2): 162-163.

Sharma, B. R., K. V. Rao, K. P. R. Vittal, Y. S. Ramakrishna, and U. Amarasingh, 2010: Estimating the potential of rainfed agriculture in India: Prospects for water productivity improvements. Agric. Water Manage, 97: 23-30.

Siddig AMA, Adam KlI, Bahar AH, Hassan 2013. The effect of sowing date and variety on growth and yield of pearl millet (Pennisetum glaucum 1.) grown on two soil types under rain - fed condition at Zalingei Area in Sudan. Journal of Science and Technology. 2013; 3(4).

Upadhyay, PN., Dixit, A.G., Patel, J.R. and Chavda, J.R. (2001). Response of summer pearl millet to time and method of planting, age of seedling and phosphorus grown on loamy sand soils of Gujarat. Indian J. Agron. 46(1): 126130.

Uzoma AO, Eze PC, Alabi M, Mgbonu K, Aboje JE, Osunde AO. 2010.The effect of variety and planting date on the growth and yield of pearl millet in the Southern Guinea Savanna Zone of Nigeria. Journal of Agriculture and Veterinary Sciences. 2010; 2: 122-127.

Witt, M.D. 1996. Delayed planting 
opportunities with winter wheat in the central great plains. J. Prod. Agric. 9: 74-78.

Yang, J., Zhang, J., Wang, Z., Zhu, Q. and Liu, L. (2001). Water deficit-induced senescence and its relationship to the remobilization of pre-stored carbon in wheat during grain filling. Agronomy Journal. 93: 196-206.

\section{How to cite this article:}

Londhe, V. M., S. G. Birajdar, V. T. Jadhav, J. D. Jadhav and Amrutsagar, V. M. 2020. Effect of Weather Parameters on Pearl Millet (Pennisetum glaucum L.) Cultivars under Different Sowing Windows. Int.J.Curr.Microbiol.App.Sci. 9(09): 1190-1202.

doi: https://doi.org/10.20546/ijcmas.2020.909.147 